\title{
Probing the functional properties of mammalian dendrites (R. Llinas and M. Sugimori, J. Physiology, 1980, 305:197-213)
}

\author{
Jørn Hounsgaard • Michael Häusser
}

Published online: 20 June 2012

(C) Springer Science+Business Media, LLC 2012

\section{Introduction by Jørn Hounsgaard}

Sometimes, a paper becomes a classic for more than one reason. In the case of the paper from the Journal of Physiology by Rodolfo Llinás and Mutsuyuki Sugimori featured in this Cerebellar Classics [5], a triad of factors contributed, each of which would easily have made a classic on its own. The paper was almost born a

\author{
J. Hounsgaard $(\square)$ \\ Department of Neuroscience and Pharmacology, \\ The Panum Institute, \\ 3 Blegdamsvej, \\ 2200 Copenhagen N, Denmark \\ e-mail: jhou@sund.ku.dk \\ URL: http://inf.ku.dk

\section{Häusser ( $\square)$} \\ Wolfson Institute for Biomedical Research, \\ University College London, \\ Gower Street, \\ London WC1E 6BT, UK \\ e-mail: m.hausser@ucl.ac.uk \\ Section Editors:

\section{E. Haines} \\ Department of Anatomy, University of Mississippi Medical center, \\ 2500 North State Street, Jackson, MS 39216, USA \\ e-mail: dhaines@umc.edu

\section{M.-U. Manto} \\ FNRS-Laboratorie de Neurologie Expérimentale, \\ Université Libre de Bruxelles, ULB-Erasme, \\ Route de Lennik 808, 1070 Brussels, Belgium \\ e-mail: mmanto@ulb.ac.be
}

classic. It crowned a 10-year effort to establish dendritic electrotonus as a fundamental nonlinear property of postsynaptic integration and at the same time, initiated the idea of $\mathrm{Ca}^{2+}$ as the universal link between electrical and chemical signaling in dendrites. Finally, this and the preceding accompanying paper [4] provided the manual for how cell specific somadendritic nonlinear response properties and their underlying ionic mechanisms were to be characterized in vertebrate neurons.

Electrotonus The idea of electrically active dendrites in Purkinje cells originated from strong but indirect evidence obtained with extracellular recordings and was supported by intradendritic recordings in vivo from Purkinje cells in the cerebellum of the alligator $[2,6]$. However, with the firepower of the slice technique, the featured CEREBELLAR CLASSICS by Llinás and Sugimori [5] once and for all elevated local regenerative dendritic responses from the realm of curiosities in sharks, alligators, and birds to fundamental properties of all Purkinje cells including those in mammals. The paper also changed the general perception of active response properties in dendrites. From a narrow vehicle for aberrant dendritic spike generation in response to synaptic excitation dendritic response properties emerged as intrinsic pattern generators that provide astonishing complexity and dynamics to the translation of synaptic input in dendrites into output spike patterns in axons. The experimental findings and the discussion also made the paper instrumental in bridging a gap that had developed between vertebrate and invertebrate cellular neurophysiology. 
Ca signaling The paper did not just confirm that dendritic spikes in mammalian Purkinje cells were generated at multiple "hot-spots" were graded by conductance and were mediated by $\mathrm{Ca}^{2+}$ as shown previously in nonmammalian Purkinje cells [1]. With the unseen mechanical stability offered by the slice technique, highquality intracellular recordings from dendrites could be maintained literally for hours. This made it possible to demonstrate and scrutinize the existence of $\mathrm{Ca}^{2+}$ spikes and $\mathrm{Ca}^{2+}$ plateau potentials and analyze the different abilities of these conductances to recruit $\mathrm{Ca}^{2+}$ - and voltage-dependent $\mathrm{K}^{+}$conductances. The concept of the dendritic tree as a multicompartment communication system of local $\mathrm{Ca}^{2+}$ signaling domains-modulated by intrinsic $\mathrm{K}^{+}$conductances as well as inhibitory synaptic conductances - was finally proved beyond doubt. Hence, the paper paved the way for the studies of $\mathrm{Ca}^{2+}$ signaling and metabotropic regulation that were to follow. Interestingly, the outright discussion of intracellular $\mathrm{Ca}^{2+}$ signaling that figure strongly in a preceding review paper also by Llinás and Sugimori [3] is conspicuously absent in the rather dry and factual discussion in the CEREBELLAR CLASSICS featured here.

Slicework The two papers on the electrophysiological properties of somata and dendrites of mammalian Purkinje cells changed the status of the slice technique in electrophysiology. From a marginal position of as a specialized tool for exploration of transmitter pharmacology, brain slices suddenly became the main source of new ideas on the role of neurons in brain function! In the years that followed, the papers served as templates for characterizing the response properties of inferior olivary neurons, thalamic neurons as well as pyramidal cells and interneurons in the cerebral cortex and the hippocampus. Passive dendrites soon belonged to the past even in spinal motoneurons! Eventually, whole cell patch recordings made even small neurons readily approachable. Supplemented with imaging techniques and transgenic technology slices have provided the means to explore and analyze $\mathrm{Ca}$ signaling and metabotropic modulation in real time with high spatial resolution. Ironically, integrating this emerging wealth of knowledge on cellular properties and signaling into functional understanding at the network level has become a new challenging bottle neck only to be addressed in vivo.

\section{A Personal Perspective by Michael Häusser}

I read the paper by Llinás and Sugimori with great enthusiasm as an undergraduate student, and still continue to recommend it to my own students with the same enthusiasm many years later. This study, together with the companion paper on somatic recordings published in the same issue, transformed our understanding of active dendrites and continues to have a durable impact on the field. It appeared at a time when the field of dendritic research was still in its infancy: due to their inaccessibility, the importance of dendrites was underestimated by most neuroscientists for many years; and the lack of first-rate electrophysiological data left many unanswered questions and stimulated vigorous debate. In particular, the concept of active dendrites, though it had been explored theoretically by Rall a decade before [7], lacked direct experimental support. The Llinás and Sugimori paper not only provided a detailed description of dendritic excitability and a clear picture of the underlying biophysical mechanisms, but also gave us the equivalent of a map - the celebrated schematic in Fig. 4 which ended up in many textbooks.

The masterful electrophysiological technique demonstrated in the paper had a major impact on me and on many others in the field, setting a new "gold standard" for dendritic recordings. Before Llinás and Sugimori embarked on their study, the few dendritic recordings that had been made were done in vivo, typically using extracellular recordings and/or in nonmammalian preparations $[7,8]$. In the late 1970s, slice preparations - which today are the mainstay of neurophysiology labs around the world - were only starting to become accepted as a promising model system for studying neuronal excitability and synaptic transmission. Llinás and Sugimori adapted previous methods for preparing cerebellar slices and added an important component: the use of Hoffmann contrast modulation optics, which allowed the dendrites of individual Purkinje cells to be visualized and targeted for microelectrode recordings. This crucially allowed them to make recordings from identified locations in the dendritic tree, a prerequisite for the "map" in Fig. 4. The stability offered by the slice preparation also permitted the long-duration recordings necessary for the detailed characterization of dendritic physiology, as well as the pharmacological experiments required for dissecting the underlying biophysical mechanisms. Even today, the quality of the recordings in the paper remains a benchmark, and the electrophysiological signature of dendritic excitability illustrated in the figures has 'iconic' status for anyone studying Purkinje cell physiology.

But the beautiful recordings performed by Llinás and Sugimori were not merely illustrative: their experiments directly addressed and resolved several key controversies in the field. First, Llinás and Sugimori resolved the longstanding question of whether dendrites are electrically excitable enough to generate spikes. While spikes had been recorded from dendrites before, it was unclear where these spikes were initiated. The fact that there was a clear spatial segregation of spike types in Purkinje cells - sodium action 
potentials at the soma, and calcium spikes in the distal dendrites - provided convincing evidence that distal dendrites can generate spikes autonomously from the soma. This was later confirmed with the advent of simultaneous double patch-clamp recordings from the soma and dendrites of many cell types [9], showing that dendritic spikes can be generated before or in complete isolation of axonal action potentials. Second, the distinct biophysical basis of the somatic and dendritic spike types provided the first indication of non-uniform distribution of voltage-gated conductances in the somatodendritic membrane. This was revealed by pharmacological dissection of the underlying conductances - with TTX-sensitive sodium channels shown to underlie the somatic action potentials, while dendritic spikes were TTX-insensitive, and mediated by calcium channel activation. This implied that the respective channel types (and possibly also other channels crucial for shaping the spike, such as potassium channels) must also have a non-uniform distribution in the dendritic tree. Direct confirmation of this idea was only possible much later, by using patch-clamp recordings to map voltage-gated channel distributions, which showed that Purkinje cell dendrites essentially lack dendritic sodium channels [10]. Dendritic gradients in the distribution of voltage-gated channels have since been demonstrated in many cell types in the mammalian brain. Third, the study provided one of the first clear indications that dendrites generate functional compartmentalization of electrical signals. Llinás and Sugimori showed that the somatic action potentials fail to backpropagate effectively into the dendrites; while dendritic calcium spikes in turn do not propagate faithfully to the soma, remaining confined to the distal dendrites. Thus, different subcellular compartments experience different electrical signals; allowing local processing of synaptic input. These findings formed the basis of a large body of subsequent experimental and theoretical work exploring the idea that dendrites could provide independent processing compartments, enhancing the computational power of the neuron $[9,11]$.

The principles illuminated by the Llinás and Sugimori paper - the ability of dendrites to generate autonomous spikes; the underlying non-uniform distribution of voltagegated conductances; and the functional compartmentalization imparted by the dendritic tree- - have been taken up and further probed by many subsequent investigators of dendritic function. Indeed, it would be hard to deny that the paper has underpinned and inspired much of the research into dendritic function in the three decades since its publication. While it was not the first study to record from dendrites in slices [12], or to intracellularly record dendritic spikes [12, 2], the stunning quality of the recordings together with the favorable properties of the Purkinje cell dendrites allowed Llinás and Sugimori to reveal new principles and present them in a remarkably clear, forceful manner. In the intervening years, it is hardly surprising that the introduction of new techniques has led to some details of their conclusions being modified, such as those regarding the origin of spontaneous firing and plateau potentials (which have been shown to be driven primarily by axonal sodium channels, and in particular the resurgent sodium current [13]), and that the expression of calcium channels is not restricted to the dendrites. But the quality of their recordings, the importance of the findings and the clarity of the presentation ensure that the landmark study by Llinás and Sugimori will continue to guide and inspire students of dendritic function - and their mentors-for generations to come.

Acknowledgments The paper by Llinás and Sugimori is reprinted with the permission of the publishers and editor of the Journal of Physiology. The editors greatly appreciate this courtesy.

\section{References}

1. Llinás R, Hess R. Tetrodotoxin-resistant dendritic spikes in avian Purkinje cells. Proc Natl Acad Sci U S A. 1976;73(7):2520-3.

2. Llinás R, Nicholson C. Electrophysiological properties of dendrites and somata in alligator Purkinje cells. J Neurophysiol. 1971;34(4):532-51.

3. Llinás R, Sugimori M. Calcium conductances in Purkinje cell dendrites: their role in development and integration. Prog Brain Res. 1979;51:323-34.

4. Llinás R, Sugimori M. Electrophysiological properties of in vitro Purkinje cell somata in mammalian cerebellar slices. J Physiol. 1980;305:171-95.

5. Llinás R, Sugimori M. Electrophysiological properties of in vitro Purkinje cell dendrites in mammalian cerebellar slices. J Physiol. 1980;305:197-213.

6. Nicholson C, Llinás R. Field potentials in the alligator cerebellum and theory of their relationship to Purkinje cell dendritic spikes. J Neurophysiol. 1971;34(4):509-31.

7. Rall W, Shepherd GM. Theoretical reconstruction of field potentials and dendrodendritic synaptic interactions in olfactory bulb. J Neurophysiol. 1968;3:884-915.

8. Llinás R, Nicholson C, Freeman JA, Hillman DE. Dendritic spikes and their inhibition in alligator Purkinje cells. Science. 1968;160:1132-5.

9. Häusser M, Spruston N, Stuart GJ. Diversity and dynamics of dendritic signaling. Science. 2000;290:739-44.

10. Stuart G, Häusser M. Initiation and spread of sodium action potentials in cerebellar Purkinje cells. Neuron. 1994;13:703-12.

11. Mel BW. Synaptic integration in an excitable dendritic tree. J Neurophys. 1993;70:1086-101.

12. Wong RK, Prince DA, Basbaum AI. Intradendritic recordings from hippocampal neurons. Proc Natl Acad Sci U S A. 1979;76:986-90.

13. Raman IM, Bean BP. Resurgent sodium current and action potential formation in dissociated cerebellar Purkinje neurons. J Neurosci Off J Soc Neurosci. 1997;17:4517-26. 


\title{
ELECTROPHYSIOLOGICAL PROPERTIES OF IN VITRO PURKINJE CELI DENDRITES IN MAMMALIAN CEREBELLAR SLICES
}

\author{
BY R. LLINÁS AND M. SUGIMORI \\ From the Department of Physiology and Biophysics, \\ New York University Medical Center, 550 First Avenue, \\ New York, NY 10016, U.S.A.
}

(Received 14 August 1979)

SUMMARY

1. Intradendritic recordings from Purkinje cells in vitro indicate that white matter stimulation produces large synaptic responses by the activation of the climbing fibre afferent, but antidromic potentials do not actively invade the dendritic tree.

2. Climbing fibre responses may be reversed in a manner similar to that observed at the somatic level. However, the reversal does not show the biphasicity often seen at somatic level.

3. Input resistance of these dendrites was found to range from 15 to $30 \mathrm{M} \Omega$. The non-linear properties seen at the somatic level for depolarizing currents are also encountered here. However, there seems to be less anomalous rectification.

4. Detailed analysis of repetitive firing of Purkinje cells elicited by outward DC current shows that, as in the case of the antidromic invasion, the fast somatic potentials (s.s.) do not invade the dendrite actively. However, the dendritic spike bursts (d.s.b.s) interposed between the s.s. potentials are most prominent at dendritic level.

5. Two types of voltage-dependent Ca responses were observed. At low stimulus level a plateau-like depolarization is accompanied by a prominent conductance change; further depolarization produces large dendritic action potentials. These two classes of response are TTX-resistant but are blocked by Cd, Co, Mn or D600, or by the removal of extracellular $\mathrm{Ca}$.

6. Following blockage of the $\mathrm{Ca}$ conductance, plateau potentials produced by a non-inactivating $\mathrm{Na}$ conductance are observed mainly near the soma indicating that this voltage-dependent conductance is probably associated with the somatic membrane.

7. Spontaneous firing in Purkinje cell dendrites is very similar to that observed at the soma. However, the amplitude of these bursts is larger at dendritic level. It is further concluded that these TTX-insensitive spikes are generated at multiple sites along the dendritic tree.

8. Six ionic conductances seem to be involved in Purkinje cell electroresponsiveness: $(a)$ an inactivating and $(b)$ a non-inactivating $\mathrm{Na}$ conductance at or near the soma, (c) a spike- and $(d)$ a plateau-generating Ca conductance, and (e) voltagedependent and $(f)$ Ca-dependent $K$ currents.

9. The possible role of these conductances in Purkinje cell integration is discussed.

0022-3751/80/8850-0456 \$07.50 (C) 1980 The Physiological Society 


\section{INTRODUCTION}

Although experimental evidence obtained from neurones in the central nervous system indicates that dendrites of many cells have passive membrane properties (Jack, Noble \& Tsien, 1975), it is now well documented that in some neurones, dendrites are capable of active electroresponsiveness. Among these chromatolysed motoneurones (Eccles, Libet \& Young, 1958), Purkinje cells (Llinás \& Nicholson, 1971) and hippocampal pyramidal cells (Spencer \& Kandel, 1961; Anderson \& Lømo, 1966; Schwartzkroin \& Slawsky, 1977) clearly demonstrate such properties. Furthermore, several lines of evidence indicate that dendritic electroresponsiveness differs from that encountered in the soma or the axon on at least two counts: $(a)$ dendritic action potentials are conducted in a non-continuous manner (Llinás \& Nicholson, 1971; Llinás \& Hess, 1976; Wong, Prince \& Basbaum, 1979) and (b) dendritic currents seem to be generated by voltage-dependent membrane conductance to $\mathrm{Na}$ and, more prominently, to Ca (Barrett \& Barrett, 1976; Llinás \& Hess, 1976; Llinás, Sugimori \& Walton, 1977; Llinás \& Sugimori, 1978; Schwartzkroin \& Slawsky, 1977; Wong, Prince \& Basbaum, 1979; Yarom \& Llinás, 1979; Llinás \& Yarom, 1980).

Indeed dendritic responses seem to be produced by the summation of several distinct all-or-none components, indicating a non-uniform distribution of active membrane properties over the dendritic tree. In fact, multiple sites of membrane electroresponsiveness separated by portions of non-excitable membrane are, formally, the most likely distribution of ionic conductances capable of generating non-continuous conduction (Traub \& Llinás, 1979; Pellionisz, 1979; Dodge, 1979). In the present set of experiments, intradendritic recording has allowed the study of the passive and active characteristics of Purkinje cell dendrites at different levels in the dendritic arbour, as well as the ionic requirements for such dendritic conductances.

\section{METHODS}

The procedures used to implement the cerebellar slice technique are the same as reported in the accompanying paper (Llinás \& Sugimori, 1980). The precise localization of the recording site in the dendritic tree was determined under direct vision using Hoffman modulation optics (Hoffman, 1977) and confirmed by the injection of fast green dye. In many instances, however, while such injections gave the exact location of dendritic impalement, the rapid movement of this dye away from the site of injection made it difficult to isolate the recording site photographically in a clear enough manner to allow non-colour reproduction. The micro-electrodes utilized were very similar to those employed for intrasomatic recording. For a detailed description of the techniques, see Llinás \& Sugimori (1980).

\section{RESULTS}

Intradendritic recording from a Purkinje cell in the cerebellar slice preparation may be obtained for up to $24 \mathrm{hr}$ after isolation. Using modulation optics, dendritic segments may often be seen as they arise from the Purkinje cell soma and throughout the course of their approach to the surface of the molecular layer. More often, however, the penetration is performed blindly and the exact level of dendritic recording is indicated by the micro-electrode position with respect to the Purkinje cell layer. 
As several types of processes may be impaled in the molecular layer (somata and dendrites of basket and stellate cells, dendrites of Golgi and Bergmann glial cells), three general criteria can be utilized to identify Purkinje cell dendrites. First, injection of fast green dye will stain the Purkinje cell dendritic tree as well as the body of origin of this dendritic arbour. Secondly, stimulation of the underlying white matter often produces a small antidromic action potential whose amplitude

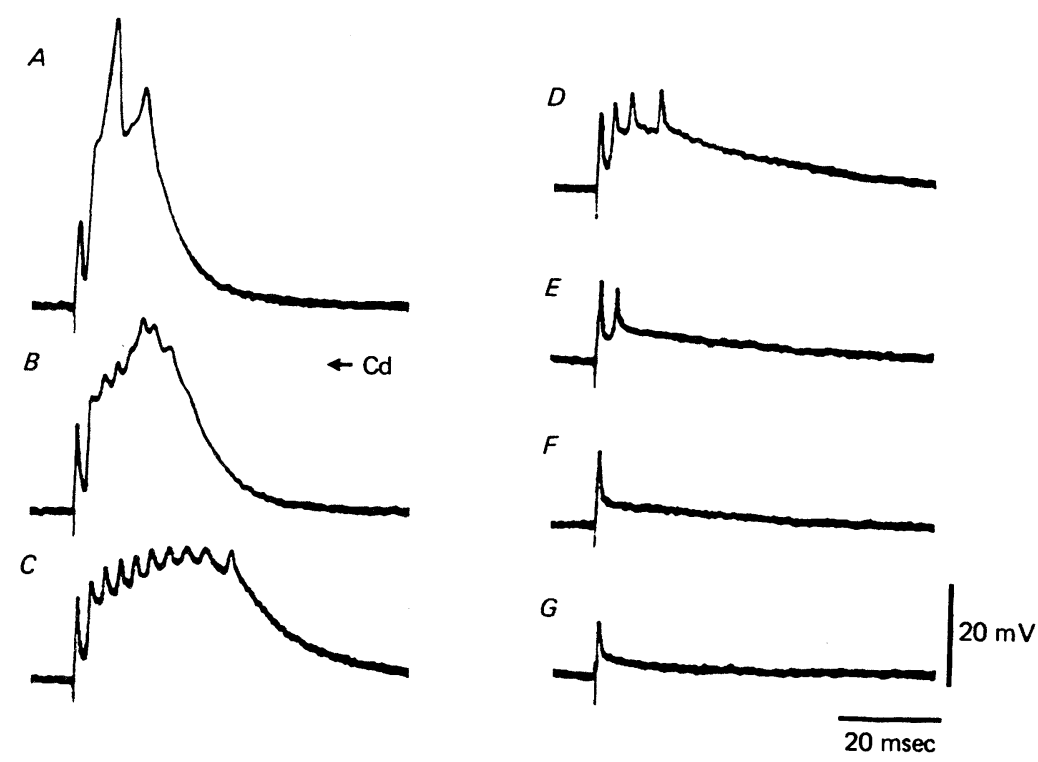

Fig. 1. Antidromic and synaptic-evoked potentials recorded intradendritically from a Purkinje cell. $A$, white matter stimulation generates an early sharp depolarization followed by a prolonged all-or-none synaptic response produced by activation of the climbing fibre. $B-G$, addition of $\mathrm{Cd}$ to the bath leads to a reduction of the late potential unmasking fast spikes. $F$ and $G$, only the antidromic invasion of the Purkinje cell soma remains and is electrotonically conducted to the dendrite.

varies with the exact level of dendritic penetration (Fig. 1). Thirdly, this antidromic invasion is generally followed by an all-or-none orthodromic potential produced by the activation of the climbing fibre-Purkinje cell synapse (Fig. 1). These three criteria were utilized throughout this study to characterize intradendritic Purkinje cell recordings. Direct observation with fast green dye injection indicates that stable sites of intradendritic recording generally correspond to large diameter dendrites. The recording sites often occurred at or near bifurcation points where the dendrites may have a diameter of up to $10 \mu \mathrm{m}$, even at segments located rather superficially in the molecular layer.

\section{Climbing fibre activation of Purkinje cell dendrites}

As shown in Fig. $1 \mathrm{~A}$, the all-or-none synaptic depolarization produced by the climbing fibre afferent at the dendritic level differed from that recorded under similar conditions in the soma. In the dendrite, climbing fibre activation produced an all-or-none excitatory post-synaptic potential which, in turn, generated a set of 
action potentials having several peaks and a time course much slower than that observed for the fast somatic spike (s.s.) potentials.

The synaptic origin of this response was demonstrated in two ways: first, by showing its dependence on the presence of Ca conductance and secondly by its reversal following post-synaptic membrane depolarization (Fig. 2). Indeed, addition
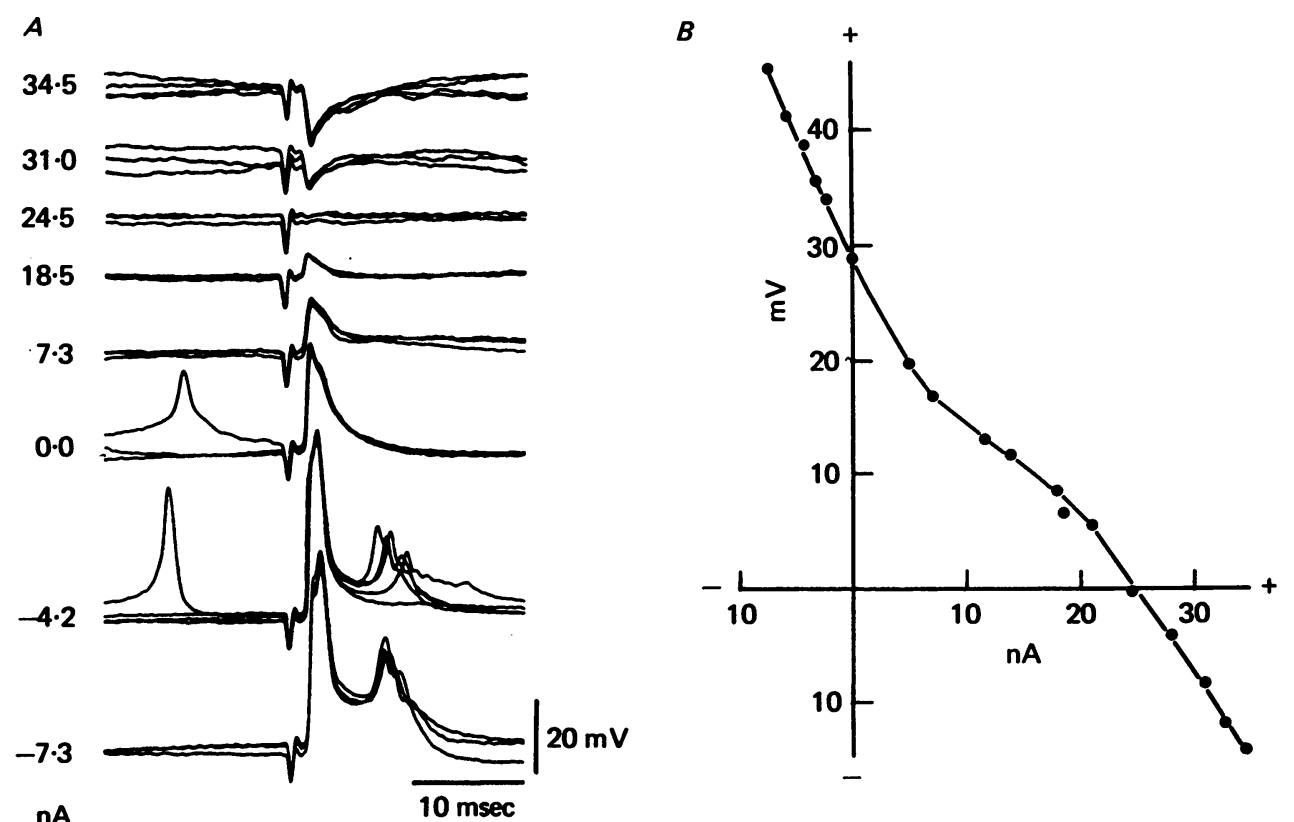

Fig. 2. Reversal of dendritic climbing fibre-evoked e.p.s.p. $A$, synaptic potential produced by direct stimulation of the climbing fibre increases in amplitude with hyperpolarizing current of $4 \cdot 2$ and $7 \cdot 3 \mathrm{nA}$ magnitude. A decrease and eventually a reversal of this potential is observed with depolarizing currents from $7 \cdot 3$ to $34.5 \mathrm{nA}$. Note the close-to-monophasic nature of the reversal. The plot in $B$ gives relationship between current and e.p.s.p. amplitude.

of $\mathrm{CdCl}_{2}$ (known to block Ca conductance and hence transmitter release [Hagiwara, 1973; Kostyuk \& Krishtal, 1977; Llinás, Steinberg \& Walton, 1976]) produced, as shown in Fig. $1 B-F$, a gradual decrease of this post-synaptic response. As the amplitude of this response decreased, the number of s.s. potentials conducted electrotonically from the soma (as will be seen in more detail below) increased as the postsynaptic potential subsided, unmasking a set of s.s. potentials. Thus, 6 min after the addition of $\mathrm{Cd}$ (Fig. $1 C$ ), climbing fibre activation generated ten s.s. responses. This activation further decreased and was finally reduced to a single antidromic spike in Fig. $1 G$ indicating, as shown in the accompanying paper (Llinás \& Sugimori, 1980 ), that the antidromic activation of this neurone was not altered by $\mathrm{CdCl}_{2}$ while synaptic transmission and the dendritic Ca electroresponsiveness were clearly blocked.

A more direct demonstration of the synaptic nature of this response is seen in Fig. $2 A$. This set of dendritic records was obtained proximal to those shown in Fig. 1 (approximately $100 \mu \mathrm{m}$ from the Purkinje cell layer). Here, as the membrane 
was hyperpolarized from rest $(0 \cdot 0 \mathrm{nA})$ by inward $\mathrm{DC}$ current injection, the climbing fibre activation became larger as did the secondary spikes which it generated. Depolarization of the membrane first reduced the climbing fibre-generated e.p.s.p. $(7 \cdot 5-18.5 \mathrm{nA})$ and as the current injection was increased further, a reversal of the
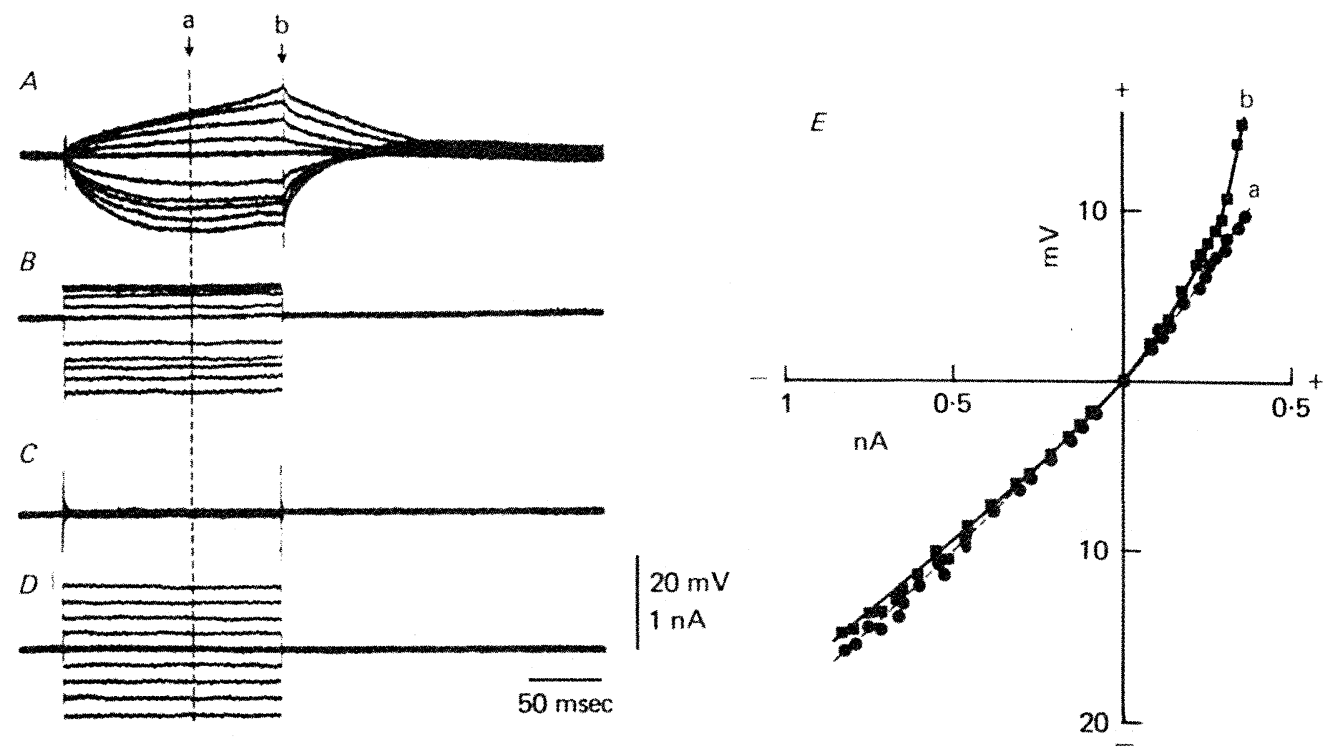

Fig. 3. Dendritic input resistance. $A$, potentials recorded during outward and inward current pulse injection (currents are illustrated in $B$ ). $C$ and $D$, as in $A$ and $B$ but with the electrode immediately outside the cell. $E$, plot of the current-voltage relationship. $\mathrm{a}$ and $\mathrm{b}$ correspond to points used in the plot in $E$.

TABLE 1. Values for resting potential, spike height and input resistance from five Purkinje cell dendrites

\begin{tabular}{|c|c|c|c|}
\hline Experiment no. & $\begin{array}{c}\text { Resting } \\
\text { potential } \\
(\mathrm{mV})\end{array}$ & $\begin{array}{c}\text { Calcium* } \\
\text { spike height } \\
(\mathrm{mV})\end{array}$ & $\begin{array}{c}\text { Input } \dagger \\
\text { resistance } \\
(\mathrm{M} \Omega)\end{array}$ \\
\hline $25-1$ & 58 & 38 & 15 \\
\hline 25-2 & 55 & 35 & 25 \\
\hline 25-3 & 57 & 50 & 18 \\
\hline 25-4 & 65 & 60 & 30 \\
\hline 26-2 & 60 & 40 & 17 \\
\hline Mean \pm S.D. & $59+3 \cdot 4$ & $44 \cdot 6 \pm 9 \cdot 2$ & $21 \pm 5 \cdot 6$ \\
\hline
\end{tabular}

* Spike heights were measured from the resting potential level to the peak of the spike.

$\dagger$ Input resistances were determined with the hyperpolarizing current pulses $(0.5 \mathrm{nA})$ in order to avoid delayed rectification.

all-or-none synaptic potential ensued at $31 \cdot 0-34.5 \mathrm{nA}$. Note that at $24.5 \mathrm{nA}$ the biphasicity of the e.p.s.p. reversal obtained at this dendritic location was less marked than those seen at the somatic level. The plot of the current-voltage relationship for this response (shown to the right) indicates a close-to-monotonic relation, resembling that obtained at the soma (Llinás \& Sugimori, 1980). 


\section{Input resistance}

Input resistance was determined by injection of square current pulses through the recording micro-electrode. One such set of records is seen in Fig. 3. $A$ and $B$ show the current and the voltage recordings respectively, while $C$ and $D$ are controls obtained extracellularly for similar current pulses in the immediate vicinity of the intracellular recording site. $E$ is a plot of the current-voltage relationship. Measurements

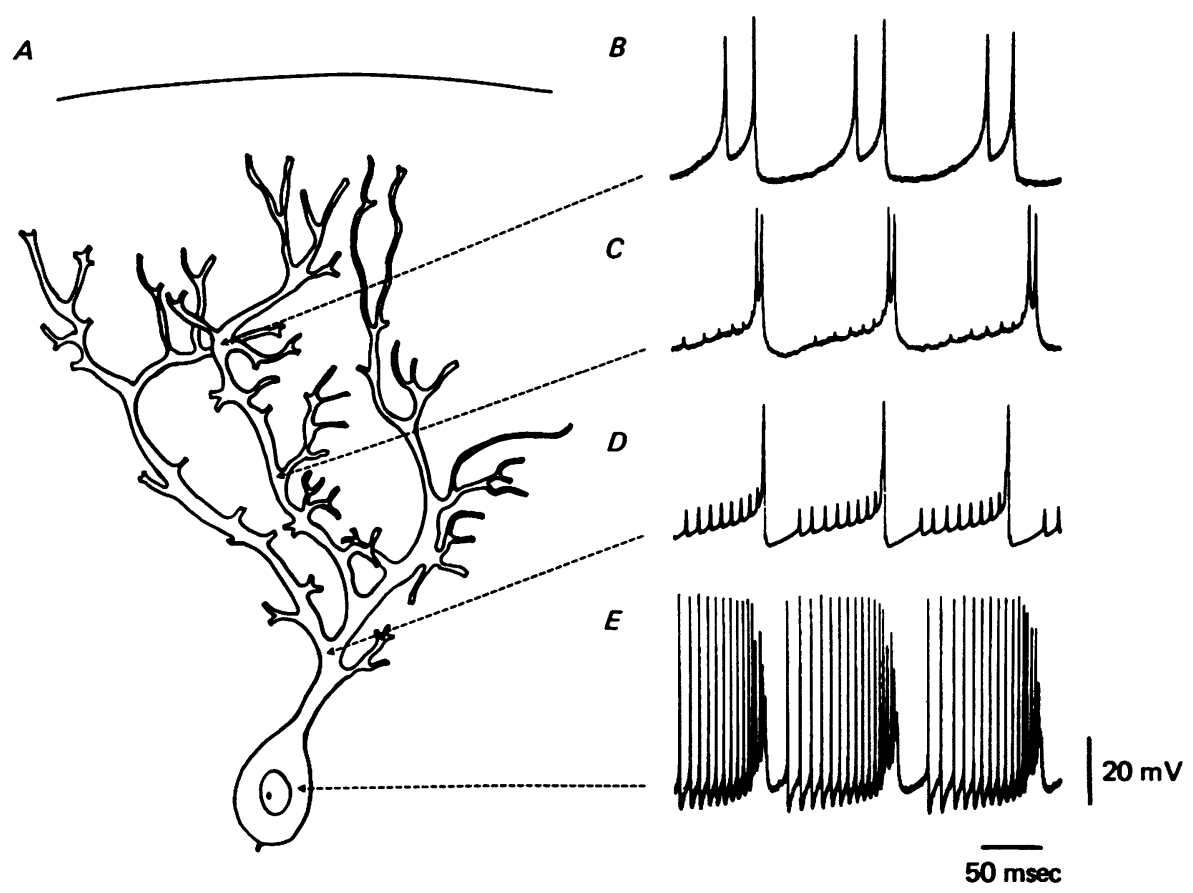

Fig. 4. Composite picture showing the relationship between somatic and dendritic action potentials following DC depolarization through the recording electrode. A clear shift in amplitude of the s.s. against the dendritic Ca-dependent potentials is seen when comparing the more superficial recording in $B$ with the somatic recording in $E$. Note that at increasing distances from the soma the fast spikes are reduced in amplitude and are barely noticeable in the more peripheral recordings. However, the prolonged and slowrising burst spikes are more prominent at dendritic level.

were made at $A$, a and $\mathrm{b}$, and a close-to-linear relationship was seen in the hyperpolarizing direction at these two points. Non-linearity was, however, found with depolarizing pulses. This is especially evident in measurements made at the end of the current injection pulse $(E, \mathrm{~b})$. Table 1 gives measurements of the input resistance encountered in five dendritic impalements.

\section{Direct DC stimulation of dendrites at different levels fom the Purkinje cell layer}

In order to describe more clearly the electrical properties of Purkinje cell dendrites, a composite picture of intradendritic recordings obtained at different dendritic levels, in a set of Purkinje cells, is shown in Fig. 4. The drawing to the left was made from a camera-lucida reconstruction of a neurone injected with horseradish peroxidase (HRP). The points of impalement represent accurately the distances between the 
Purkinje cell layer and the site of recording. However, since such recordings could not be obtained in a single cell, the points of recording simply indicate the most probable site of penetration given the level of the impalement. The purpose of the illustration is to indicate that direct DC stimulation (on the order of $1 \mathrm{nA}$ ) through the recording electrode produced, as shown for the somatic recording, two kinds of action potentials: a fast spike which has been characterized as generated by a voltagedependent $\mathrm{Na}$ conductance change (Llinás \& Nicholson, 1971; Llinás \& Sugimori, 1978) and a slower Ca-dependent spike. As the recordings were made at increasing distances from the soma, the amplitude of the Na-dependent fast somatic spikes (Fig. $4 E$ ) was reduced such that in the upper third $(B)$ of the dendritic tree they are hardly seen. These records demonstrate that the Na-dependent s.s. potentials are passively conducted towards the dendrites as initially observed in lower vertebrates (Llinás \& Nicholson, 1971). On the other hand, the Ca-dependent action potentials were large in the more superficial dendrites and quite small in the soma, indicating a change in emphasis in the dendrites from a Na-dominated to a Ca-dominated voltage-dependent membrane conductance.

\section{Ionic conductances generating dendritic spikes}

\section{Calcium-dependent potentials}

Plateau responses. A more detailed study of dendritic electroresponsiveness at mid-dendritic level is seen in Fig. 5. Here graded depolarizing currents of low magnitude can generate all-or-none depolarizations which outlast the duration of the stimulus. These responses have constant amplitude and may last for hundreds of milliseconds. Invariably in neurones showing appropriate resting potential $(60 \mathrm{mV}$ and up) and resistance (no less than $10 \mathrm{M} \Omega$ ), somatic action potentials can be observed upon this plateau-potential (Fig. $5 \mathrm{~A}$ ) when the recording is below middendritic level. As in the case of the non-inactivating Na plateaus seen in the soma (Llinás \& Sugimori, 1980), these plateaus are accompanied by a large conductance increase. However, in this particular case, the plateau is Ca-dependent, as will be seen below. If the current pulse is prolonged beyond that shown in Fig. $5 \mathrm{~A}$, in addition to the plateau a burst of slow action potentials accompanied by an increase in the firing frequency of the somatic Na spikes is generally obtained (Fig. $5 B$ ).

Further increase in the duration of the transdendritic depolarization generates s.s. potentials interrupted by prolonged bursts of large action potentials (Fig. $5 C$ ). This type of repetitive discharge was not significantly modified by addition of TTX $10^{-5} \mathrm{~g} / \mathrm{ml}$. to the bath (Fig. $5 D$ ). Rather, as expected from the results described by Llinás \& Sugimori (1980), the small s.s. potentials seen in $A-C$ were completely blocked while the large spike bursts and the hyperpolarization which followed showed very little modification.

A more detailed illustration of these TTX-insensitive spikes is shown in Fig. 6. In this set of records, short outward current pulses across the dendritic membrane produced, in the presence of TTX, self-regenerating depolarizing plateaus of similar amplitude but different durations. While the amplitude of the plateau for a given recording site was constant, the amplitude varied from 10 to $30 \mathrm{mV}$ from one recording to the next, the larger potentials occurring in the more peripheral recording 
sites. Often the plateaus were high enough to activate dendritic spike burst (d.s.b.) potentials.

That these TTX-insensitive spike bursts are produced by the summation of several all-or-none components is indicated by Fig. $6 B$. Here a portion of the uppermost trace in $A$ (bracket with arrows) is displayed at a faster sweep speed and at a slightly larger gain. The slow onset of the dendritic spikes and a multiplicity of spike com-

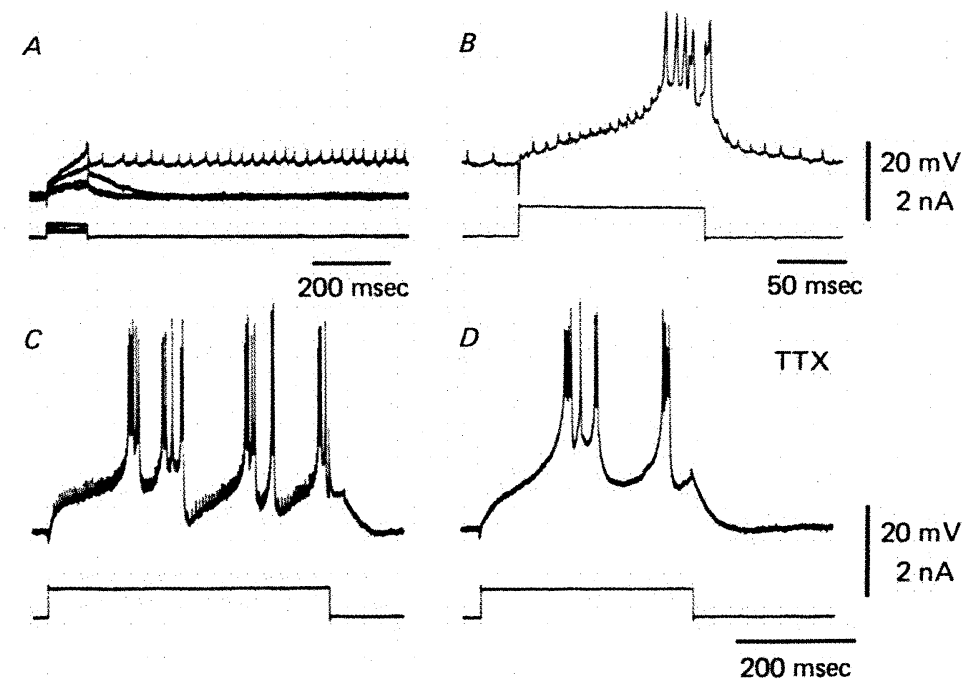

Fig. 5. Two types of Ca-dependent spiking. $A$, intradendritic stimulation generates a local response which becomes a prolonged plateau on which s.s. potentials are seen. This plateau depolarization may last for several seconds. $B$, a further increase in the amplitude and duration of the stimulus generates a burst of spikes. $C$, further increases in the duration of the stimulus pulse produces a set of dendritic spike bursts interposed by small somatic potentials. $D$, addition of TTX to the bath has little or no effect on the voltage-dependent dendritic bursts.

ponents (especially in the latter response) are clearly visible and resemble the multipeak bursts seen in Figs. 1, 2, 4 and 5. Note that the falling of these action potentials is much faster than their rising phase. Replacement of $\mathrm{Ca}$ by $\mathrm{Cd}$ in the Krebs-Ringer solution produces in $C$ the blockage of both the plateau and the action potentials, indicating that these two types of voltage-dependent responses are $\mathrm{Ca}$-dependent.

\section{Non-inactivating $\mathrm{Na}$ potentials}

As described by Llinás \& Sugimori (1980), removal of Ca from the bath or the introduction of $\mathrm{Cd}, \mathrm{Co}, \mathrm{Mn}$ or D600 produced a complete blocking of most dendritic electroresponsiveness. However, in preparations where TTX-insensitive spikes had not been previously blocked, nor $\mathrm{Na}$ removed from the medium, depolarizing pulses evoked prolonged all-or-none plateau-potentials, similar to those seen at the somatic level (Fig. $7 \mathrm{~A}$ ). These types of response were generally observed in the basal dendrites near the soma. As in the case of the non-inactivating $\mathrm{Na}$ potentials recorded in the soma, these plateau-potentials could be blocked with TTX (Fig. $7 B$ ). Because of their spatial distribution and the smaller amplitude shown at the dendritic level, it seems probable that this $\mathrm{Na}$ current is prevalent in the somatic region. 


\section{Spontaneous firing}

Another form of activity encountered in intradendritic recordings is shown in Fig. $8 \mathrm{~A}$. Here trains of dendritic spike bursts were seen regularly every $15 \mathrm{sec}$, each train lasting approximately 10 sec. Such activity was generally seen during the first hours after slice isolation but could be observed in slices for at least $20 \mathrm{hr}$
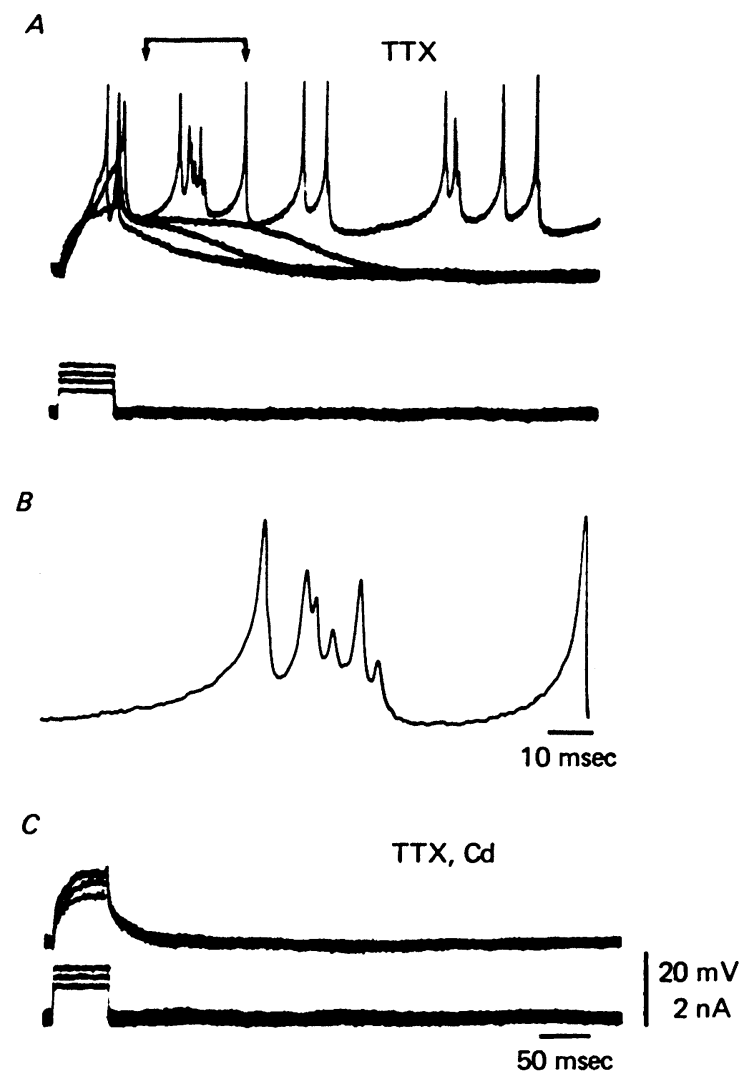

Fig. 6. Calcium-dependent plateau and burst spikes. Details of the plateau potentials seen intradendritically with short depolarizations in the presence of TTX are shown. As the stimulus is increased, prolonged local responses are seen which ultimately result in the large plateau potential upon which dendritic spike bursts are superimposed. $B$, detail of one of these bursts is shown at high sweep speed to demonstrate the noncontinuous nature of this spike conduction, indicating multiple sites of spike origin. $C$, addition of $\mathrm{CdCl}_{2}$ to the TTX solution produces a complete blockage of the plateau and the burst response recorded intradendritically.

following isolation. Since this type of bursting was also seen extracellularly under conditions of minimal compression or damage, its presence does not seem related to injury caused by dendritic impalement. In Fig. $8 B$ a single burst train is shown at faster sweep speed. Note that during this prolonged burst train the individual d.s.b.s were not uniform; rather, at the beginning their frequency was slightly higher and their amplitude fluctuated. At the end of the train, as the membrane potential became 


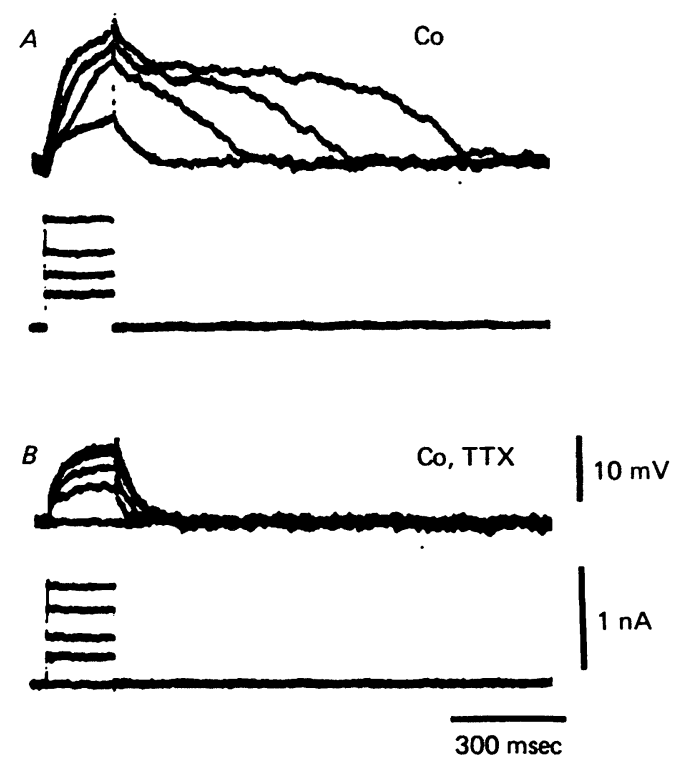

Fig. 7. Intradendritic recording following addition of Co to the bath. This recording was obtained in the primary dendrite approximately $100 \mu \mathrm{m}$ from the soma. $A$, direct depolarization produces prolonged plateau responses in the absence of fast somatic firing. $B$, addition of TTX to the bath blocks the plateau potential.

hyperpolarized, their frequency decreased and the individual d.s.b. consisted of two to three spikes, each burst separated by a clearly visible after-hyperpolarization. Note, however, that their amplitude continued to fluctuate. In $C$, application of TTX to the bath produced virtually no change in the burst train activity which in this particular case occurred immediately after a depolarizing pulse.
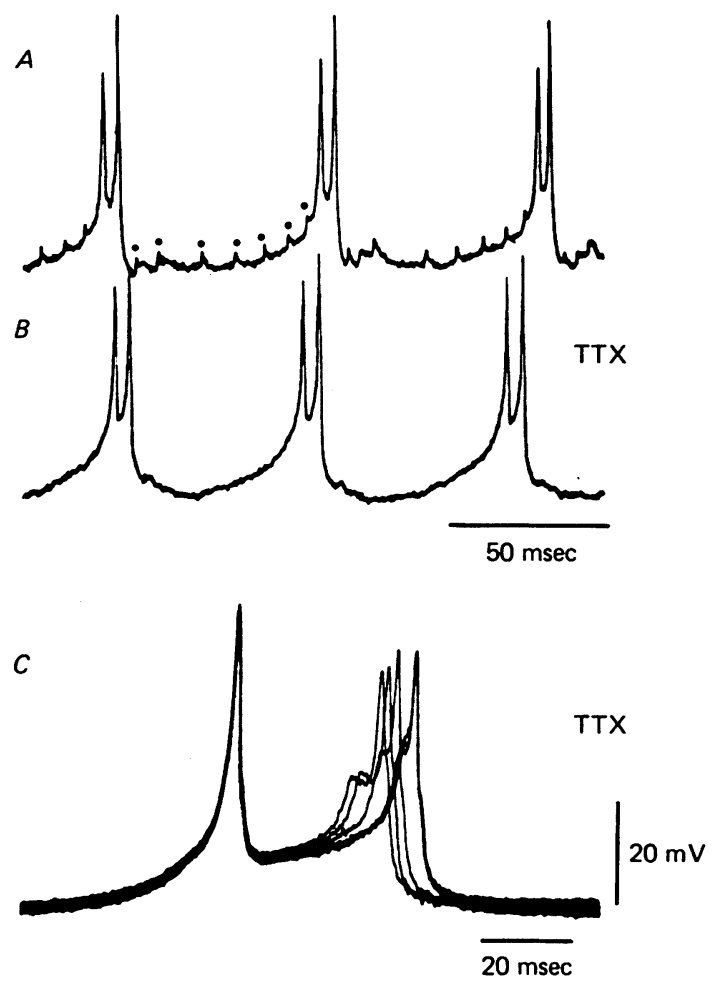

Fig. 9. Spontaneous dendritic bursting. $A$, dendritic bursting is seen to be accompanied by s.s. potentials (dots). $B$, in the presence of TTX, s.s. potentials are blocked but the basic character of dendritic bursting remains. $C$, superimposed bursts at higher sweep speed show the rather slow onset of the Ca-dependent action potential and of the spike which follows. This second spike is seen to be composed of several components, indicating non-continuous conduction.

In short then, these spontaneous d.s.b. trains seen in dendrites seem to be largely induced by Ca currents. A clear demonstration of this may be seen in Fig. $9 A$ and $B$ where the d.s.b. and s.s. are shown at higher sweep speed before and after TTX, demonstrating once again that only the s.s. potentials were blocked by this drug.

Fig. 8. Spontaneous bursting observed immediately after impalement. $A$, spike bursting demonstrating a period of about $25 \mathrm{sec}$ consisting of a train of spike bursts lasting approximately $10 \mathrm{sec}$ and a period of quiescence lasting approximately $15 \mathrm{sec}$. $B$, the frequency of the dendritic spikes varies such that in the beginning the frequency is high but becomes low near the end. On the average, the frequency of the spike burst was approximately $10-20 /$ sec. $C$, addition of TTX produces no significant change in the frequency of over-all characteristics of the prolonged spike train. 
Since removal of the Ca current (by absence of extracellular Ca or addition of Cd, Co, $\mathrm{Mn}$ or D600) abolished both the d.s.b. and the slower membrane potential fluctuations which ultimately activate the d.s.b. trains, it is concluded that the burst trains are generated by prolonged $\mathrm{Ca}$ conductance change. Whether this change is produced solely by a voltage-dependent Ca conductance modulated by a subsequent Ca-dependent $K$ conductance, or whether in addition some of this membrane potential modulation is controlled by a long-term Ca-dependent electrogenic process, is not clear at this juncture. Besides the obvious possibility of an electrogenic metabolic pump, the possibility of a long-acting transmitter or a peptide substance being released during this bursting activity must be kept in mind (cf. Llinás, 1979).

\section{DISCUSSION}

The obvious advantages of the mammalian cerebellar slice preparation over the in vivo approach are especially evident in studying the functional properties of Purkinje cell dendrites. The very fact that in the in vitro system accurate determination may be made of the precise location of the recording micro-electrode represents in itself an enormous technical advantage over other neuronal systems in the mammalian brain. In addition, the fact that the climbing fibre-Purkinje cell synapse can be studied at intradendritic level and that the synaptic potentials may be reversed will no doubt prove useful in future studies of central synaptic transmission as well as of the Ca electroresponsiveness which is part of this response at dendritic level.

The data presented above address several points pertaining to dendritic electrophysiology, the most salient of which are the ionic requirements for dendritic spiking.

\section{Ionic basis of dendritic spiking}

The present set of recordings suggests that the ion mainly responsible for dendritic excitability is Ca. Moreover, direct stimulation at dendritic level has revealed two different types of electroresponsiveness phenomena: $(a)$ a slow plateau-like depolarization which may last for hundreds of milliseconds and $(b)$ d.s.b.s which represent an intermediate state between the plateau potential and the fast action potentials seen at somatic level.

These two types of dendritic response have several properties in common. First, they are most prominent and have their lowest threshold at dendritic level. Secondly, they are TTX-insensitive and, thirdly, they are blocked by ions which block Ca conductance and are abolished in the absence of extracellular Ca. There are, on the other hand, distinct differences between them which will be described in detail below.

\section{Calcium plateau potentials}

These calcium plateau potentials are all-or-none responses which show little inactivation. Thus, given a threshold stimulus (threshold is generally about $10 \mathrm{mV}$ ), the plateau response may last from a few to several hundred milliseconds. In this in vitro condition, the level of the plateau shows little variation in amplitude for a given recording site, and the input reistance is reduced markedly, as in the case of 
the Na-dependent plateau at somatic level. However, the amplitude of the plateau potential fluctuated from 10 to $30 \mathrm{mV}$, depending on the site of dendritic impalement. At the more proximal dendritic level, the amplitude was usually smaller than in the more peripheral dendrites. The range of amplitude does suggest an equilibrium state between, at least, $\mathrm{Ca}$ and $\mathrm{K}$ potentials. Since $\boldsymbol{E}_{\mathrm{Ca}}$ should drive the potential to a more positive value, the $K$ conductance is probably high and may be triggered by a combined voltage- and Ca-dependent mechanism.

\section{Dendritic spike bursts}

The d.s.b.s are characterized by their rather slow onset, their large amplitude at dendritic level which fluctuated between 30 and $60 \mathrm{mV}$, and their multiple spike components.

As illustrated in Fig. 9, the time course of these action potentials was much longer than that of the $\mathrm{Na}$ spike. The rise time was particularly slow and had a concave trajectory. These d.s.b.s always terminated in a clear after-hyperpolarization probably generated by voltage- and Ca-dependent $\mathrm{K}$ conductances. While it is clear that bursting in Purkinje cells is most often observed in vivo following climbing fibre activation, such bursting can also be observed under other conditions (Eccles Llinás \& Sasaki, 1966).

Although the threshold for these d.s.b.s is apparently high when activated from the soma, their threshold at dendritic level is much lower. As seen in Figs. 5 and 6, current depolarizations in the vicinity of $10 \mathrm{mV}$ were often sufficient to generate them. In contrast, as seen in the accompanying paper (Llinás \& Sugimori, 1980), depolarizations in the order of $20 \mathrm{mV}$ were required at the somatic level to evoke d.s.b.s.

The spatial distribution of these bursts is such that the extracellular inward currents are most clearly seen at dendritic level from 100 to approximately $300 \mu \mathrm{m}$ from the surface (Llinás \& Hess, 1976), i.e. they comprise most of the dendritic tree length. In addition, measurement of extracellular Ca concentration following local stimulation (known to generate Ca spikes; Llinás \& Hess, 1976) or during spreading depression clearly indicates inward Ca movement at Purkinje cell dendritic level (Nicholson, Bruggencate, Stoeckle \& Steinberg, 1978).

Another point to be noted with respect to these recordings is that, as seen in vivo, (Llinás \& Nicholson, $1969 ; 1971$; Llinás \& Hess, 1976) and in vitro in other preparations (Schwartzkroin \& Slawsky, 1977; Wong et al. 1979; Yarom \& Llinás, 1979; Llinás \& Yarom, 1980), calcium dendritic spikes demonstrate many sites of origin. Thus, during spike bursting, action potentials tend to have several different components very much as was found in the in vivo preparation. Unfortunately, the activation of the parallel fibre system (which allows a very restricted and controlled dendritic stimulation, ideal for inducing peripheral dendritic spikes) cannot be utilized here. Nevertheless, the phenomenology observed in the slices is in complete accordance with that found in similar circumstances in the in vivo situation. Another point that makes this particular preparation less fortunate for studying the integrative properties of Ca spiking in dendrites is the absence of stellate and basket cell inhibition. This is especially so since dendritic input seems to be able to block d.s.b. potentials in a selective manner (Llinás \& Nicholson, 1971; Wong et al. 1979). 
In short then, d.s.b.s appear to be generated at different points in the dendritic tree, appear to be Ca-dependent, and are followed by a prolonged and powerful $\mathrm{K}$ conductance change. Whether d.s.b.s and Ca plateaus are fundamentally different electroresponsive properties or whether they are simply different in the magnitude of the Ca current being evoked is yet to be determined. Nevertheless, the voltagedependent conductance may be similar to the so-called Ca conductance seen in other preparations (Baker, Hodgkin \& Ridgway, 1971). In fact, a theoretical model for the hippocampal pyramidal cells (Traub \& Llinás, 1979) based on a kinetic model obtained for Ca conductance from the presynaptic fibre of the squid ganglion (Llinás et al. 1976), has yielded results similar to those reported here.

\section{Sodium conductance in dendrites}

The possibility that dendrites may generate $\mathrm{Na}$ action potentials was tested in several ways. Indeed, as seen in Figs. 4 and 5, Na spikes were not actively generated in dendrites following antidromic stimulation, climbing fibre activation or direct depolarization through the recording pipette. Furthermore, when recording at different distances from the Purkinje cell body, the invasion by the $\mathrm{Na}$ spikes disappeared almost totally at about two-thirds of the dendritic length (Fig. 4). The possibility that such lack of antidromic invasion is due to impalement damage seems to be ruled out by extracellular field studies (Llinás \& Nicholson, 1969; Nicholson \& Llinás, 1971). Those results indicate a similar phenomenology in vivo even in the presence of a superimposed dendritic depolarization generated by the background parallel fibre activity. This is not surprising given that the action current encounters a rather large load produced by the extensive ramification of the Purkinje cell dendrite. However, the fact that neither local stimulation nor climbing fibre activation can produce larger amplitude spikes at the dendritic level is a strong indication that the lack of invasion is not due to the small magnitude of the antidromic action current, but rather to the lack of a voltage-dependent $\mathrm{Na}$ conductance in the dendrites. Another aspect of dendritic excitability relates to the possible presence of a non-inactivating $\mathrm{Na}$ current. Thus, as seen in Fig. 7 following blockage of the $\mathrm{Ca}$ conductance, at the dendritic level a prolonged TTX-sensitive Na-dependent action potential is elicited by direct dendritic stimulation. For the most part, however, these potentials were observed in the lower dendritic tree and usually their amplitude, as indicated in Fig. 8, was much smaller then their counterparts at the somatic level. Obviously the same ambiguities which were considered for the distribution of Ca must be taken into account in analysing this potential. On the other hand, since the potential was always smaller at the dendritic level, a tentative conclusion is that a non-inactivating $\mathrm{Na}$ current must be more significant at the somatic level.

\section{Spontaneous firing}

The results presented here are consistent with the view that, as in other neurones (Wong \& Prince, 1978; Llinás \& Yarom, 1980), the autorhythmicity is predominantly Ca-dependent and is basically a dendritic property. While several mechanisms for its genesis may be considered, this oscillation is clearly related to a set of sequential activations: first a voltage-dependent $\mathrm{Ca}$ conductance which generates a stepdepolarization of the membrane potential for a protracted period. This depolarization 
generates $\mathrm{Ca}$-dependent dendritic firing followed by a $\mathrm{Ca}$ and voltage-dependent $\mathrm{K}$ conductance. This latter would generate the falling phase of the Ca spikes and ultimately the termination of the dendritic burst trains. However, the mechanism for the quiescent period between burst trains seems to be different from that generating the falling phase and after-hyperpolarization of the dendritic Ca spike. The two main reasons for this conclusion are that $(a)$ the time course of the quiescent period was indeed much longer and its level more negative than that seen between the burst spikes and, more importantly, $(b)$ the membrane conductance was higher at the after-hyperpolarization than during the quiescent period. Indeed, while many possibilities may be considered, the most attractive one is that of an active electrogenic transport as seen following repetitive activation in other neuronal systems (see Thomas, 1972).

\section{Integrative properties of Purkinje cells}

Contrasting the electrophysiological properties of Purkinje cells recorded at somatic level with those obtained at dendritic level, a picture emerges suggesting a basic difference between the excitable properties of the somatic and dendritic regions of the Purkinje cell. The soma seems to have a sharp voltage-dependent $\mathrm{Na}$ conductance change which generates the fast somatic spikes. These potentials are translated into action potentials that are orthodromically conducted down the axon (Ito \& Simpson, 1971) and are clearly limited to the soma and initial segment since they seem not to contribute directly to dendritic excitability. In addition, the soma seems to have an important voltage-dependent non-inactivating slow $\mathrm{Na}$ current which has a lower threshold for activity than the action potential itself and apparently dictates the firing properties of these neurones, which resemble the 'class-2' (Hodgkin, 1948) repetitive firing characteristic of crab nerve. This slow $\mathrm{Na}$ conductance is obviously capable of maintaining a certain level of somatic depolarization for a protracted period and may be quite important in translating slow dendritic events into axonal action potentials. A similar plateau modulation of membrane excitability could also be seen at the dendritic level; however, in this case the potential seemed to be related more to a Ca conductance. In the in vivo state, graded plateau responses are probably masked by the parallel fibre input which regulates membrane potential continuously and, thus, regulates the Ca- and voltagedependent $K$ conductance. The observation that following either the removal of $\mathrm{Ca}$ or the blockage of the Ca current, Purkinje cells developed very prolonged trains of somatic firing and may depolarize even to the level of inactivation, further suggests that this slow Ca-K system represents a most important modulator of membrane excitability. In addition to modifying the level of membrane potential, this $\mathrm{Ca}-\mathrm{K}$ system, and indeed the slow $\mathrm{Na}$ system, are capable of long-term modification of the input resistance of the cell. This implies that the 'cable properties' of these neurones are not constant but, rather, are continuously modified by the non-linearities introduced by these prolonged electroresponsive properties.

Thanks are due our colleagues, C. Nicholson, B. Rudy and K. Walton, for their comments on this paper. Research was supported by United States Public Health Service program grant NS-13742 from the National Institute of Neurological and Communicative Disorders ond Stroke. 


\section{REFERENCES}

Andersen, P. \& Lomo, T. (1966). Mode of activation of hippocampal pyramidal cells by excitatory synapses on dendrites. Expl Brain Res. 2, 247-260.

Baker, P. F., Hodgkin, A. L. \& Ridgway, E. G. (1971). Depolarization and calcium entry in squid giant axons. $J$. Physiol. 218, 709-755.

BARRETT, E. F. \& BARRETT, J. N. (1976). Separation of two voltage-sensitive potassium currents. and demonstration of a tetrodotoxin-resistant calcium current in frog motoneurones. $J$. Physiol. 255, 737-774.

DoDGe, F. A. (1979). The nonuniform excitability of central neurons as exemplified by a model of the spinal motoneuron. In The Neurosciences: Fourth Study Program, ed. SснмiтT, F. O. \& Worden, F. G., pp. 439-455. Cambridge, Mass.: M.I.T. Press.

Eccles, J. C., Libet, B. \& Young, R. R. (1958). The behaviour of chromatolysed motoneurones studied by intracellular recording. $J$. Physiol. 143, 11-40.

Eccles, J. C., Llinás, R. \& SASAKI, K. (1966). Intracellularly recorded responses of the cerebellar Purkinje cells. Expl Brain Res. 1, 161-183.

Hagiwara, S. (1973). Calcium spikes. Adv. Biophys. 4, 71-102.

Hodgkin, A. L. (1948). The local electric changes associated with repetitive action in a nonmedullated axon. J. Physiol. 107, 165-181.

Hoffman, R. (1977). The modulatory control microscope: principle and performance. $J$. Microsc. 110, 205-222.

Iто, M. \& Simpson, J. I. (1971). Discharges in Purkinje cell axons during climbing fiber activation. Brain Res. 31, 215-219.

JACk, J. J. B., Noble, D. \& Tsien, R.W. (1975). Electric Current Flow in Excitable Cells. Oxford: Clarendon Press.

Kostyuk, P. G. \& Krishtal, O. A. (1977). Separation of sodium and calcium currents in the somatic membrane of mollusc neurones. $J$. Physiol. 270, 545-568.

LLINAs, R. (1979). The role of calcium in neuronal function. In The Neurosciences: Fourth Study Program, ed. Schmitt, F. O. \& Worden, F. G., pp. 555-571. Cambridge, Mass.: M.I.'I. Press.

LlinÁs, R. \& Hess, R. (1976). Tetrodotoxin-resistant dendritic spikes in avian Purkinje cells. Proc. natn. Acad. Sci. U.S.A. 73, 2520-2523.

LLINÁs, R. \& NrCholson, C. (1969). Electrophysiological analysis of alligator cerebellar cortex : a study on dendritic spikes. In Neurobiology of Cerebellar Evolution and Development, ed. Llinás, R., pp. 431-465. Chicago: American Medical Association.

Luinas, R. \& Nicholson, C. (1971). Electrophysiological properties of dendrites and somata in alligator Purkinje cells. $J$. Neurophysiol. 34, 534-551.

Llinás, R., Steinberg, I. Z. \& Walton, K. (1976). Presynaptic calcium currents and their relation to synaptic transmission: voltage clamp study in squid giant synapse and theoretical model for the calcium gate. Proc. natn. Acad. Sci. U.S.A. 73, 2918-2922.

Lurnas, R. \& Sugrmori, M. (1978). Dendritic calcium spiking in mammalian Purkinje cells: in vitro study of its function and development. Neirosci. Abstr. 4, 66.

LlinÁs, R. \& Sugimori, M. (1980). Electrophysiological properties of in vitro Purkinje cell somata in mammalian cerebellar slices. J. Physiol. 305, 171-195.

Llinás, R., Sugimori, M. \& Walton, K. (1977). Calcium dependent spikes in the mammalian Purkinje cells. Neurosci. Abstr. 3, 58.

LliNás, R. \& YAROM, Y. (1980). Electrophysiological properties of mammalian inferior olivary cells in vitro. In The Inferior Olivary Nucleus, ed. Courville, J. New York: Raven Press.

Nicholson, C., Bruggencate, G. ten, Stoeckle, H. \& Steinberg, R. (1978). Calcium and potassium changes in extracellular microenvironment of cat cerebellar cortex. J. Neurophysiol. 41, 1026-1039.

Nicholson, C. \& Llinas, R. (1971). Field potentials in the alligator cerebellum and theory of their relationship to Purkinje cell dendritic spikes. $J$. Neurophysiol. 34, 509-513.

Pellionisz, A. (1979). Modeling of neurons and neuronal networks. In The Neurosciences: Fourth Study Program, ed. Sснмгтт, F. O. \& Worden, F. G., pp. 525-546. Cambridge, Mass.: M.I.T. Press.

Schwartzkroin, P. A. \& Slawsky, M. (1977). Probable calcium spikes in hippocampal neurons. Brain Res. 135, 157-161. 
SPENCER, W. A. \& KANDEx, E. R. (1961). Electrophysiology of hippocampal neurons. IV. Fast prepotentials. $J$. Neurophysiol. 24, 272-285.

Thomas, R. C. (1972). Electrogenic sodium pump in nerve and muscle cells. Physiol. Rev. 52, 563-594.

Traub, R. D. \& LLINAs, R. (1979). Hippocampal pyramidal cells: significance of dendritic ionic conductances for neuronal function and epileptogenesis. $J$. Neurophysiol. 42, 476-496.

Wong, R. K. S. \& Prince, D. A. (1978). Participation of calcium spikes during intrinsic burst firing in hippocampal neurons. Brain Res. 159, 385-390.

Wong, R. K. S., Prince, D. A. \& Basbaum, A. I. (1979). Intradendritic recordings from hippocampal neurons. Proc. natn. Acad. Sci. U.S.A. 76, 986-990.

YAROM, Y. \& LLINÁs, R. (1979). Electrophysiological properties of mammalian inferior olive neuron in in vitro brain stem slices and in vitro whole brain stem. Neurosci. Abstr. 5, 109. 